\title{
Correction to: Research on Vehicle Network Routing Algorithm Based on Statistical Behavior
}

Hui Li, Jun Feng, Yanli Gong, and Jialing Liu

\author{
Correction to: \\ Chapter "Research on Vehicle Network Routing Algorithm \\ Based on Statistical Behavior" in: X. Sun et al. (Eds.): \\ Artificial Intelligence and Security, CCIS 1253, \\ https://doi.org/10.1007/978-981-15-8086-4_35
}

In the originally published version of the chapter 35, the affiliation information of the authors Hui Li, Jun Feng, Yanli Gong, Jialing Liu was incorrect. The affiliation information has been corrected as School of Electronics and Automation, Inner Mongolia Electronic Information Vocational Technical College, Hohhot, 010070, Inner Mongolia, China. 